\title{
Differential replication of dengue virus serotypes 2 and 3 in coinfections of C6/36 cells and Aedes aegypti mosquitoes
}

\author{
Diana C Quintero-Gil ${ }^{1}$, Marta Ospina², Jorge E Osorio-Benitez ${ }^{3}$, Marlén Martinez-Gutierrez ${ }^{1,4}$ \\ ${ }^{1}$ Programa de Estudio y Control de Enfermedades Tropicales-PECET, Universidad de Antioquia, Medellín, \\ Colombia \\ ${ }^{2}$ Secretaria Seccional de Salud y Protección Social de Antioquia, Medellín, Colombia \\ ${ }^{3}$ Department of Pathobiological Sciences, School of Veterinary Medicine, University of Wisconsin, Madison,WI, \\ United States \\ ${ }^{4}$ Grupo de Investigación para el Fortalecimiento de la Salud y el Bienestar-GIFOSABI. Universidad Cooperativa de \\ Colombia, Bucaramanga, Colombia.
}

\begin{abstract}
Introduction: Different dengue virus (DENV) serotypes have been associated with greater epidemic potential. In turn, the increased frequency in cases of severe forms of dengue has been associated with the cocirculation of several serotypes. Because Colombia is a country with an endemic presence of all four DENV serotypes, the aim of this study was to evaluate the in vivo and in vitro replication of the DENV-2 and DENV-3 strains under individual infection and coinfection conditions.

Methodology: C6/36HT cells were infected with the two strains individually or simultaneously (coinfection). Replication capacity was evaluated by RT-qPCR, and the effects on cell viability were assessed with an MTT (3-(4, 5-dimethylthiazol-2-yl)-2,5-diphenyltetrazolium bromide) assay. Additionally, Aedes aegypti mosquitoes were artificially fed the two strains of each serotype individually or simultaneously. The viral genomes were quantified by RT-qPCR and the survival of the infected mosquitoes was compared to that of uninfected controls. Results: In single infections, three strains significantly affected $\mathrm{C} 6 / 36 \mathrm{HT}$ cell viability, but no significant differences were found in the replication capacities of the strains of the same serotype. In the in vivo infections, mosquito survival was not affected, and no significant differences in replication between strains of the same serotype were found. Finally, in coinfections, serotype 2 replicated with a thousandfold greater efficiency than serotype 3 did both in vitro and in vivo.

Conclusions: Due to the cocirculation of serotypes in endemic regions, further studies of coinfections in a natural environment would further an understanding of the transmission dynamics that affect DENV infection epidemiology.
\end{abstract}

Key words: dengue virus; replication capacity; Aedes aegypti; C6/36 cells

J Infect Dev Ctries 2014; 8(7):876-884. doi:10.3855/jidc.3978

(Received 15 July 2013 - Accepted 01 February 2014)

Copyright (C) 2014 Quintero-Gil et al. This is an open-access article distributed under the Creative Commons Attribution License, which permits unrestricted use, distribution, and reproduction in any medium, provided the original work is properly cited.

\section{Introduction}

Currently, the most important arboviral disease worldwide is caused by the dengue virus (DENV) [1]. This disease can occur in several clinical forms that are commonly known as dengue and have been classified according to the level of severity as either dengue (with or without warning signs) and severe dengue [2]. The development of severe disease forms is associated with multiple factors, some of which are related to the virus and the virulence of some strains, and others that are related to the host immune response [3]. Approximately 96 million cases of dengue infection are reported annually [4], mainly in the tropical and subtropical regions of the Asian $[5,6]$, African [7], and American [8] continents. In other words, approximately half of the global population is at risk for DENV-associated diseases.

The DENV replication cycle varies between vertebrate host cells (human primates) and invertebrate host cells (Aedes mosquitoes), and it is known that this alternation of hosts can significantly influence viral population variations [9]. Additionally, the virus must replicate in multiple tissues within the vector to infect a new susceptible host. If some viral populations fail to replicate effectively or otherwise replicate very efficiently in all vector organs that are involved in transmission, natural selection events that affect the biological efficacy can occur and thus confer greater viral variability [10].

The variability of DENV has permitted its classification into four antigenically different 
serotypes (DENV-1 to DENV-4), and the cocirculation of these four serotypes in specific geographic regions is associated with the epidemic potential of DENV [11]. This is of particular interest in Colombia, which has recorded the cocirculation of all four serotypes since 2006 [12]. In addition to this phenomenon, in Colombia, strains of the Asian genotype of DENV-2 were introduced in the 1990s; these were grouped into a new Asian-American genotype [13]. These DENV-2 strains have been associated with increased virulence [14] and have been replacing the strains of American origin [15]. Furthermore, strains of the genotype III of DENV-3 reappeared in 2001 [12]; these strains have also been associated with the presence of severe disease forms [16]. It is known that some DENV-2 genotypes might be more virulent [17], and in Colombia, these have been associated with recent epidemics [13]. DENV-3 it has also been associated with severe disease forms since its reappearance in 2001 [16].

Despite the constant circulation of all four serotypes and the increased virulence of some serotypes relative to others, there are few studies that have reported coinfection in a single individual. Coinfections have only been reported in Asian [19,20], Brazilian [20], and Peruvian [21] patients. These cases of coinfection might be related to coinfections in the mosquito vector; however, there are also few reports about mosquito coinfections, and only a few studies have demonstrated the simultaneous existence of natural serotype 2 and 3 infections in Aedes populations from Asia and America [23,24]. Additionally, the presence of intra-host competition in the vector, which may favor the replication of some viral variants over others, has been reported for other infection models, either between two serotypes of the same virus [24] or between strains of the same serotype [25], thereby favoring the transmission of one of the variants. Similar behaviors have been reported for DENV and chikungunya virus coinfections, in which the percentages of infection spread were better for the Chikungunya virus [26].

Given this background, this study evaluated whether two strains of DENV that originated from clinical isolates of the same genotype from serotypes 2 (Asian-American genotype) and 3 (genotype III), collected between 1995 and 2007 in Medellín, Colombia [27], differed with regard to their replication capacities in both an in vitro (C6/36 HT cells) and an in vivo model (Aedes aegypti) and in both single infections and coinfections.

\section{Methodology}

Viruses and cell culture

The C6/36HT cells (donated by Dr. Maria Guadalupe Guzmán of the Instituto Pedro Kourí [Pedro Kouri Institute], La Habana, Cuba) were maintained in a culture medium that was supplemented with $12 \%$ fetal bovine serum (Invitrogen, Carlsbad, CA, USA) at $34^{\circ} \mathrm{C}$ in $5 \% \mathrm{CO}_{2}$. Clinical isolates were obtained from the virus collection of the Laboratorio Departamental de Salud Publica de Antioquia [Antioquia's Public Health Laboratory] in the city of Medellin. Two DENV-2 strains previously sequenced [27], both of which belonged to the American/Asian genotype (strain DENV-2/469, isolated in 1995 and strain DENV2/3986, isolated in 2007) and two DENV-3 strains, both of which belonged to genotype III (strain DENV3/15859 GenBank accession no. FJ389908, isolated in 2002 and strain DENV-3/3832 GenBank accession no. FJ389915, isolated in 2006) were used. Viruses from the clinical isolates were stored at $-70^{\circ} \mathrm{C}$ until used.

\section{Maintenance of Aedes (Ae.) aegypti colonies}

Aedes aegypti (Rockefeller strain) mosquitoes were maintained in an insectarium at an average temperature of $28^{\circ} \mathrm{C}$ and a relative humidity of $84 \%$ with photoperiods of 12 light hours and 12 dark hours. To obtain adult specimens, larvae from the same oviposition paper were reared in plastic containers at a density of 100 larvae per liter and were fed with $1 \mathrm{mg}$ of sterile fish feed. When the individuals reached the pupal stage, they were transferred to insect breeding cages; once adults emerged, they were fed a $10 \%$ sugar solution ad libitum. The adult mosquitoes used in the experiments had emerged 7 to 10 days prior, and 50 females were placed into separate cages according to the feeding group in order to feed each of the strains to be evaluated.

Calibration curves for absolute quantification by realtime reverse transcription polymerase chain reaction (RT-PCR)

Specific segments of the C-prM regions that were obtained from the reference strains of the four DENV serotypes were cloned into the pGEM-T Easy Vector (Promega, Madison, WI, USA) according to standard protocols. Serial dilutions $\left(1 \times 10^{8}\right.$ to $1 \times 10^{3}$ genomic copies) of the plasmids with inserts for each serotype were run in real-time PCR (qPCR). The qPCR was performed using the Maxima SYBR Green qPCR Master Mix Kit (Fermentas; Thermo Scientific Inc., Waltham, MA, USA) with $2 \mu \mathrm{L}$ of RT product from a 
previous reaction and 0.5 pmol of each previously reported serotype-specific primers (mD1, mTS2, andTS3) [28], in a $20 \mu \mathrm{L}$ total reaction mixture. The amplification involved one cycle for 10 minutes at $95^{\circ} \mathrm{C}$ for polymerase activation, 35 cycles at $95^{\circ} \mathrm{C}$ for 15 seconds, $50^{\circ} \mathrm{C}$ for 15 seconds, $72^{\circ} \mathrm{C}$ for 30 seconds, and a final extension of $78.5^{\circ} \mathrm{C}$ for 30 seconds. The $\mathrm{Tm}$ curve analysis was performed following amplification to confirm the identity of the amplified product by its specific $T m$ profile; the $T m$ curve analysis included a ramp from $70^{\circ} \mathrm{C}$ to $95^{\circ} \mathrm{C}$ at a rate of $0.2^{\circ} \mathrm{C} / 5$ seconds with continuous fluorescence measurement. The amplifications were performed on a Smartcycler (Cepheid, Sunnyvale, CA, USA) system, and the $\mathrm{Ct}$ values were analyzed by linear regression to obtain serotype-specific calibration curves that were used to quantify the viral RNA.

\section{Replication capacity evaluation in C6/36 HT cells}

C6/36HT cells $\left(2.5 \times 10^{5}\right)$ were seeded into 48 -well plates. After 24 hours, the cells were infected with $2 \times 10^{5}$ genome copies $/ \mathrm{mL}$ of each of the four aforementioned strains. At 96 hours post-infection, the supernatants were collected and stored at $-70^{\circ} \mathrm{C}$ until processed to determine the amounts of viral genomes by RT-qPCR. Additionally, cell viability in the monolayers was measured by the method of metabolic reduction of 3-(4,5-dimethylthiazol-2-yl)-2,5diphenyltetrazolium bromide (MTT). Briefly, $100 \mu \mathrm{L}$ per well of MTT prepared in phosphate buffer saline was added to the cells at a final concentration of 0.5 $\mu \mathrm{g} / \mathrm{mL}$, and the cells were incubated with this compound for three hours at $34^{\circ} \mathrm{C}$ in an atmosphere of $5 \% \mathrm{CO}_{2}$. Next, $200 \mu \mathrm{L}$ of dimethyl sulfoxide (DMSO, Thermo Fisher Scientific, Rockford, USA) was added to each well, and the absorbances were read on a Varioskan Flash spectrofluorometer (Thermo Fisher Scientific, Rockford, USA Thermo Scientific) at a wavelength of $450 \mathrm{~nm}$.

\section{Replication capacity evaluation in Ae. aegypti}

Adult females were subjected to an oral challenge with each of the four virus strains mentioned previously through an artificial feeding system [29]. Each inoculum was prepared in sodium citrateanticoagulated human blood (obtained from a DENV infection-negative donor) to a final concentration of $2 \times 10^{5}$ viral genome copies $/ \mathrm{mL}$. Unfed females were selected with a manual vacuum and taken to a cage where they were anesthetized and sacrificed. Fed females were sacrificed at days $4,8,12$, and 14 postfeeding ( $\mathrm{pf}$ ). The cephalothoraxes from groups of 6 to
10 mosquitoes were harvested and individually placed into $1.5 \mathrm{~mL}$ vials that were stored at $-70^{\circ} \mathrm{C}$ until processing. Additionally, the effect of the oral challenge on the survival of mosquitoes that were fed with blood and each of the viral strains was determined. The survival of the infected mosquitoes was compared to the survival of the uninfected controls on day $14 \mathrm{pf}$, at which time the mosquitoes that had died during the course of the trial were removed in order to obtain the survival percentage relative to the total number of mosquitoes that were and were not fed the virus.

\section{Coinfections}

The strains DENV-2/3986 and DENV-3/3832 were used for the coinfections. For the in vitro assays, $2.5 \times 10^{5} \mathrm{C} 6 / 36 \mathrm{HT}$ cells were infected simultaneously with $2.5 \times 10^{5}$ genome copies $/ \mathrm{mL}$ of both strains. At 96 hours post-infection, the supernatants were collected and stored at $-70^{\circ} \mathrm{C}$ to determine the quantities of both the DENV-2 and DENV-3 strain viral genomes by RT-qPCR. In the in vivo trials, the mosquitoes were fed artificially according to the previously described protocol with a mixture of $2 \times 10^{5}$ genome copies $/ \mathrm{mL}$ of both strains (DENV-2/3986 and DENV-3/3832). At 4, 8,12 , and 14 days pf, 6 to 10 mosquitoes were sacrificed and stored in groups at $-70^{\circ} \mathrm{C}$ for viral genome quantification by RT-qPCR.

\section{Viral RNA extraction and quantification by real-time} $R T-P C R$

RNA extraction was performed on the supernatants from infected cells and the cephalothoraxes of fed females according to a modified RNAzol extraction technique [30]. Reverse transcription was performed on $0.5 \mu \mathrm{g}$ of extracted RNA. The obtained cDNA served as templates for the real-time PCR reactions that were performed with the Maxima SYBR Green qPCR Master Mix Kit (Fermentas) and the serotype-specific primers $\mathrm{mD} 1$ (DENV forward), mTS2 (DENV-2 reverse), and TS3 (DENV-3 reverse), and the reactions were cycled according to a previously described thermal profile [28]. The amplifications were performed on a Smartcycler (Cepheid) system, and the numbers of genomic copies were calculated with the previously obtained calibration curves.

\section{Statistical analysis}

At least two independent experiments with three replicates each were performed to determine the differences in the replication capacities in C6/36-HT 
cells, and a minimum of six replicates each were performed in mosquito assays. The Mann-Whitney U test was used to compare the replication capacities between two strains of the same serotype (strain DENV-2/469 versus DENV-2/3986 and strain DENV$3 / 15859$ versus DENV-3/3832). In the coinfection experiments, the genomic copy numbers were compared between the strains DENV-2/3986 versus DENV-3/3832 that were present in both the supernatants and the mosquito cephalothoraxes. All data were analyzed with SPSS for Windows version 15.0 (SPSS, Chicago, IL, USA), and differences were determined to be statistically significant if the $\mathrm{p}$ value was less than 0.05 .

\section{Results}

$D E N V-2$ and DENV-3 strain infections affected mosquito cell viability

To determine the effects of infections with two strains from serotypes 2 and 3 on C6/36HT cell viability, an MTT assay was performed at 96 hours post infection. The viability decreased significantly (Mann-Whitney U, p < 0.05) between the uninfected cultures and those infected with the strain DENV2/469 (85.7\% viability), DENV-2/3986 (72.0\% viability), or DENV-3/15859 (84.0\% viability). No significant differences (Mann-Whitney $U, p>0.05$ ) were found between the uninfected cultures and those infected with the DENV-3/3832 strain (90.1\% viability; Figure 1).

Strains of the same serotype had the same replication efficiencies in mosquito cells

To determine whether there were replication differences between the two strains of the same genotype or between serotype 2 (DENV-2/469 and DENV-2/3986) and serotype 3 (DENV-3/15859 and DENV-3/3832), C6/36HT cells were infected and the amounts of viral genome released into the supernatants were quantified at 96 hours post-infection. RT-qPCR quantification showed no statistically significant differences (Mann-Whitney U test, $\mathrm{p}>0.05$ ) between the amounts of viral RNA in the cultures infected with the strain DENV-2/469 $\left(5.05 \times 10^{8}\right.$ genome copies $\left./ \mathrm{mL}\right)$ and those infected with DENV-2/3986 $\left(9.85 \times 10^{8}\right.$ genomic copies; Figure 2A). Similarly, no statistically significant differences (Mann-Whitney $\mathrm{U}, \mathrm{p}>0.05$ ) were observed in the numbers of genomic copies produced by the cultures infected with strain DENV$3 / 15859\left(6.22 \times 10^{4}\right.$ genomic copies $)$ versus those infected with DENV-3/3832 $\left(7.46 \times 10^{4}\right.$ genomic copies; Figure 2B). However, an important difference was observed between the amounts of viral RNA in the cultures infected with DENV-2 strains, which were four logarithmic units higher than the number of copies of viral RNA from strains of serotype 3 .

Figure 1. Viability evaluation of DENV-infected C6/36HT cell cultures.

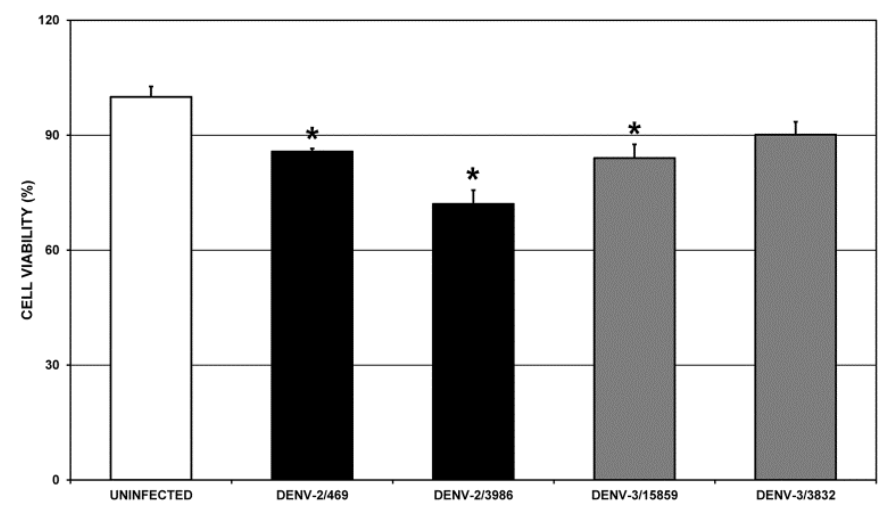

Cells were infected as indicated and the 96-hour post-infection viability was quantified with an MTT assay. Asterisks indicate statistically significant differences relative to the uninfected control (Mann-Whitney $\mathrm{U}$ test, $\mathrm{p}<0.05)$. The values are representative of two independent experiments with three replicates each $(n=6)$. Error bars represent the standard error of the mean (SEM).

Figure 2. Replication capacity evaluation of DENV-2 and DENV-3 clinical isolates in individually infected C6/36HT cells.
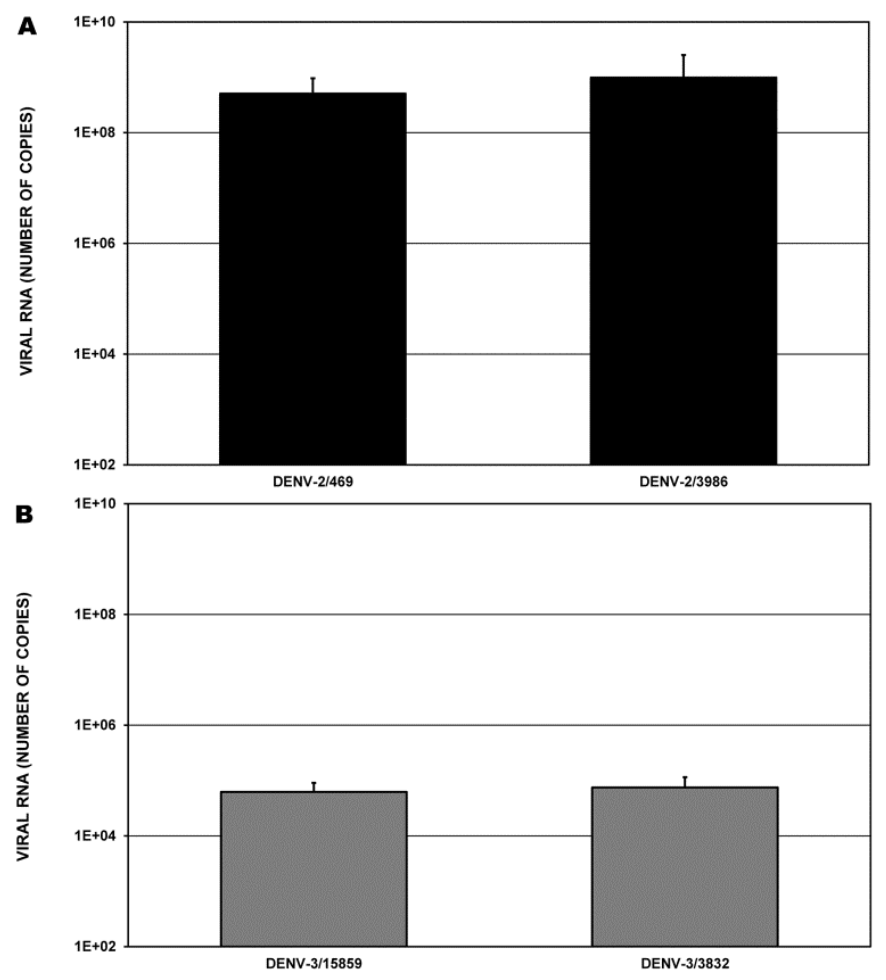

At 96 hours post-infection, viral RNA in the culture supernatants was quantified by RT-qPCR. A. DENV-2 strain-infected cells. B. DENV-3 strain-infected cells. No statistically significant differences (MannWhitney $U$ test, $p>0.05$ ) were found between the strains of the two isolates of each serotype. The values are representative of two independent experiments with three replicates each $(n=6)$. Error bars represent the standard error of the mean (SEM). 
Ae. aegypti mosquito survival is unaffected by DENV infection

Survival in the infected mosquito colonies was evaluated and compared with survival in the uninfected control colony. No statistically significant differences were observed between the survival rates of the groups fed with strains DENV-2/469 (77.9\%), DENV-2/3986 (83.5\%), DENV-3/15859 (85.0\%), or DENV-3/3832 $(79.9 \%)$ when compared to that of the uninfected control group (Student's $t$-test, $\mathrm{p}>0.05$; Figure 3).

The salivary gland dissemination efficiency was similar between strains of the same serotype

To evaluate differences in salivary gland dissemination in mosquitoes infected with the two strains of each serotype, the viral RNA amounts were quantified at different times $(4,8,12$, and 14 days post feeding). At day 12, the serotype 2 strains reached peak viral replication levels $\left(2.15 \times 10^{6}\right.$ and $2.94 \times 10^{5}$ genomic copies for strains DENV-2/469 and DENV2/3986, respectively; Figure 4A). Similarly, the viral RNA amounts for the serotype 3 strains also reached peak levels at day $12\left(3.55 \times 10^{3}\right.$ and $2.04 \times 10^{3}$ genomic copies for strains DENV-3/15859 and DENV-3/3832, respectively; Figure 4B). The replication kinetic was similar between the two strains of each serotype. Statistically significant differences were not found for the serotype 2 infections (Mann-Whitney U test, p > 0.05 ) with regard to the viral RNA amounts of the two strains at any of the time points (Figure 4A). In contrast, statistically significant differences were observed on day 8 of the serotype 3 infections (MannWhitney $U$ test, $\mathrm{p}<0.05$ ) with regard to the viral RNA amount, which was higher in DENV-3/15859 straininfected mosquitoes relative to DENV-3/3832-infected mosquitoes (Figure 4B).

Serotype 2 replicates efficiently in coinfected C6/36 HT cell cultures

To assess whether one serotype might replicate more efficiently in $\mathrm{C} 6 / 36 \mathrm{HT}$ cells, coinfections were performed with the strains DENV-2/3986 and DENV$3 / 3832$; these strains were selected because they had been isolated recently (in 2006 and 2007, respectively), and serotypes 2 and 3 had been associated with the increase of severe forms of dengue in Colombia during the last decade [13,27]. At 96 hours post-infection, serotype 2 was found to replicate more efficiently $\left(3.18 \times 10^{9}\right.$ genomic copies) than serotype $3\left(7.04 \times 10^{5}\right.$ genomic copies).
Figure 3. Survival evaluation of DENV-infected Ae. aegypti mosquitoes.

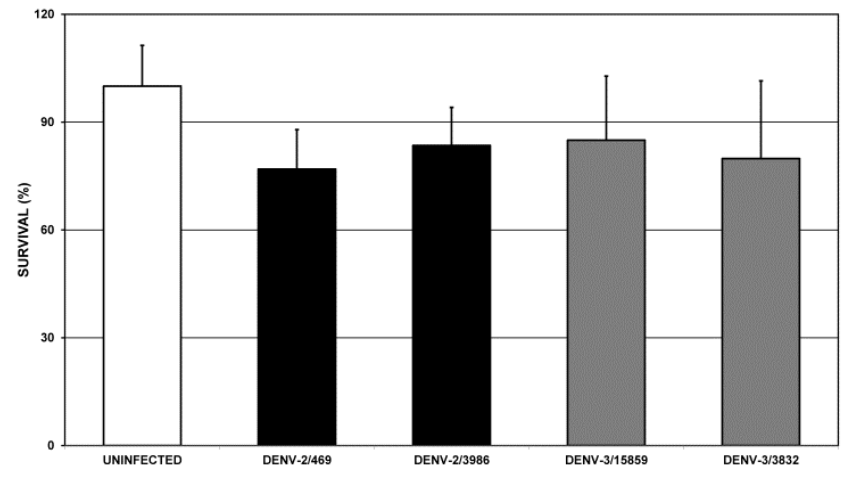

Mosquitoes were fed a mixture of blood/DENV, and the 14-day postfeeding survival rates of the infected colonies were determined. No statistically significant differences (Student's $t$-test, $\mathrm{p}>0.05$ ) were found between the survival rates of strain-infected colonies and of the uninfected control colony. The values are representative of two independent experiments for each of the experimental groups $(\mathrm{n}=19)$. Error bars represent the standard error of the mean (SEM).

Figure 4. Replication capacity evaluation of strains from the DENV-2 and DENV-3 clinical isolates in individually infected Ae. aegypti mosquitoes.
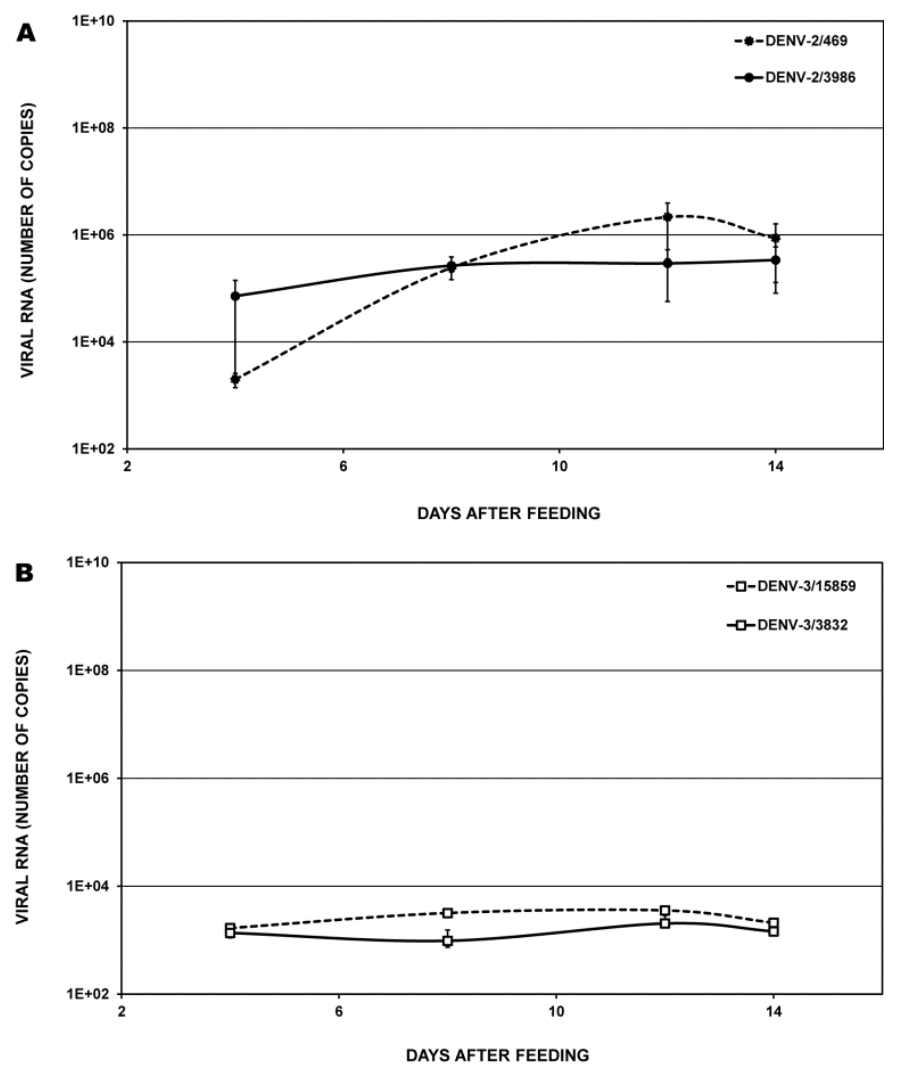

At various times post-feeding, the cephalothoraxes of individual mosquitoes were processed for total RNA extraction and subsequent viral RNA quantification by RT-qPCR. A. Mosquitoes that were fed DENV-2 strains. B. Mosquitoes that were fed DENV-3 strains. No statistically significant differences were found (Mann-Whitney $\mathrm{U}$ test, $\mathrm{p}>0.05$ ) between the two strains of each serotype. The values are representative of two independent experiments for each experimental group $(n=19)$. Error bars represent the standard error of the mean (SEM). 
Figure 5. Replication capacity evaluation of strains from the DENV-2 and DENV-3 clinical isolates in coinfected C6/36HT cells.

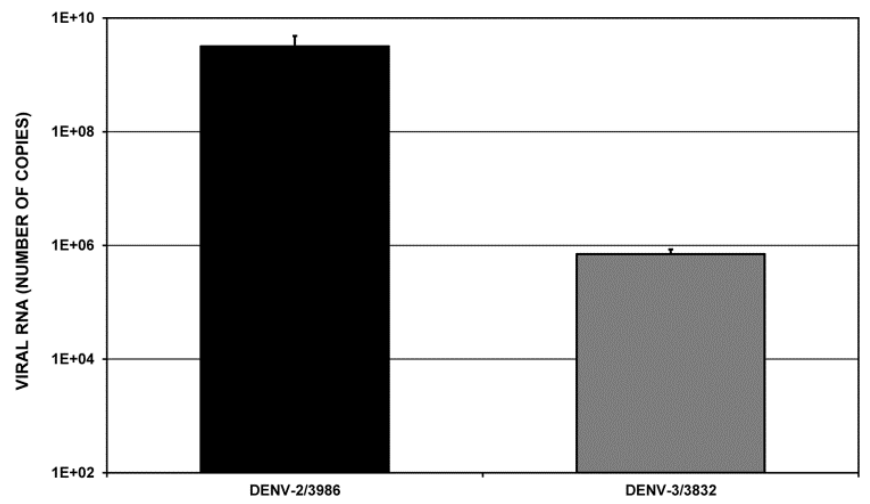

At 96 hours post-infection, the viral RNA from the coinfected culture supernatants was quantified by RT-qPCR. Serotype 2 replication was found to be significantly higher (Mann-Whitney U test, $p<0.05$ ) than serotype 3 replication. The values are representative of two independent experiments with three replicates each $(n=6)$. Error bars represent the standard error of the mean (SEM).

The number of genomic copies of serotype 2 obtained from the coinfected cultures was nearly four logarithmic units higher than the number of copies of serotype 3 from the same cultures. This difference was statistically significant (Mann-Whitney U test, p < 0.05 ; Figure 5).

Serotype 2 replicates more efficiently in coinfected Ae. aegypti

To assess whether one serotype might replicate more efficiently in Ae. aegypti mosquitoes, coinfections were performed with the strains DENV2/3986 and DENV-3/3832, and the amounts of viral RNA were quantified at different times pf $(4,8,12$, and 14 days pf). For all evaluated days, the amount of viral RNA of strain DENV-2/3986 was significantly higher than that of strain DENV-3/3832 (MannWhitney $U$ test, $p<0.05$ ). The increases in the amounts of serotype 2 viral RNA with respect to those of serotype 3 were $1.07 \times 10^{3}, 2.24 \times 10^{2}, 1.45 \times 10^{3}$, and $2.25 \times 10^{3}$ logarithmic units on days $4,8,12$, and 14 , respectively (Figure 6).

\section{Discussion}

The importance of DENV genetic variability lies not only in permitting serotype and genotype classifications, but also in enabling the associations of certain strains with their epidemic potentials. This is of particular interest in areas where all four serotypes cocirculate, such as Colombia. Additionally, this cocirculation can result in coinfections of vectors or exposed individuals, a behavior that is globally
Figure 6. Replication capacity evaluation of strains from the DENV-2 and DENV-3 clinical isolates in coinfected adult $\mathrm{Ae}$. Aegypti mosquitoes.

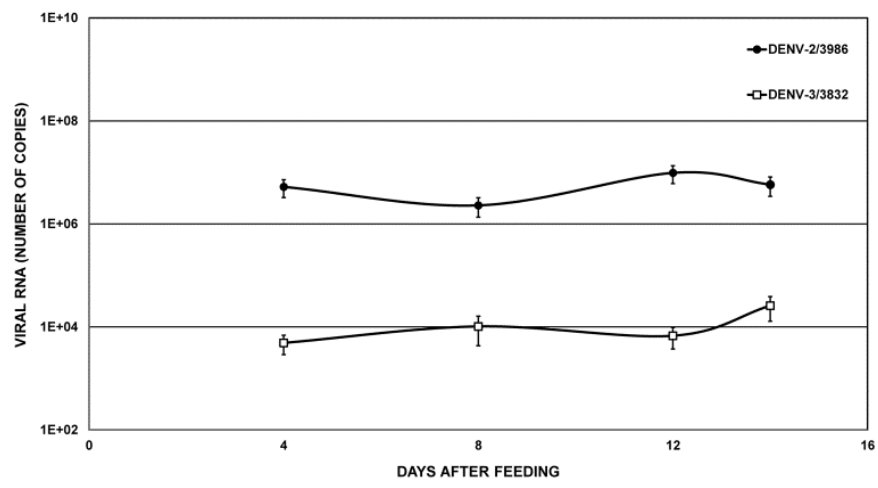

At various times post-feeding, the cephalothoraxes of individual mosquitoes were processed for total RNA extraction and subsequent viral RNA quantification by RT-qPCR. Serotype 2 replication was found to be significantly higher (Mann-Whitney $U$ test, $p<0.05$ ) than serotype 3 replication. The values are representative of two independent experiments $(n=24)$. Error bars represent the standard error of the mean (SEM).

understudied. Considering that, in recent years, an increase in the numbers of cases of dengue and severe dengue in Colombia has been linked to the circulation of more virulent serotype 2 strains [13] and the reemergence of serotype 3 strains [27], it is of particular interest to study the replication features of the strains of both serotypes. Thus in this study, we evaluated the replication capacities of strains from both serotypes 2 and 3 in both single and coinfections and in both in vitro (C6/36HT cells) and in vivo infection systems (Ae. aegypti).

During evaluations of the replication capacities of some DENV serotype 2 and 3 strains in C6/36HT cells and the effects of such replication on cell viability, it was found that two strains of the Asian-American genotype of serotype 2 (DENV-2/469 and DENV$2 / 3986$ ) decreased cell viability, but only one of the strains of serotype 3 genotype III (DENV-3/15859) had a similar effect (Figure 1), indicating differences in the biological behaviors of these strains. These results are consistent with a recent study in which strains of the same serotype but of different genotypes were reported to induce the appearance of different cytopathic effects (depending on the strain) in mosquito cells [31]. These differences might be due partly to more efficient replication of the virulent strains, and therefore might induce increased cytopathic effects and negatively affect cell viability.

No differences were found in an assessment of the replication capacities of the two viral strains of the same serotype (Figure 2), but differences were found when comparing the replication capacities of the two 
different serotypes. These results suggest that the mechanisms associated with different steps of the replication cycle are similar in strains of the same serotype but differ between serotypes. In this context, molecules that facilitate viral entry into mosquito cells have been characterized for strains of serotype 2 [32] and were found to be different from those characterized for serotype 3 [33], a phenomenon that also occurs in the infection processes of other serotypes [34]. Additionally, other studies have indicated an absence of significant differences in the viral titers obtained from $\mathrm{C} 6 / 36$ cells that were infected with different strains of serotype 2 [31,35] and 3 [31], which is consistent with the results of this study.

The effects of infection on mosquito survival (Figure 3) were first assessed in the in vivo model, and none of the strains were found to significantly affect the survival of Ae. aegypti. This might be because survival can be affected by environmental conditions and habitat rather than by the infection process. The environmental conditions that can affect survival include food availability, larval density, temperature, and endogamy [36], all conditions that were controlled during laboratory colony maintenance in the present study.

When the replication capacities were assessed in the in vivo infection model, no statistically significant differences were found in the amounts of viral genome when the two strains of the Asian-American genotype of DENV-2 were compared on different days (Figure 4A). A previous study similarly demonstrated a lack of significant differences in the binding potential of DENV to the midguts of Ae aegypti that had been infected with different serotype 2 genotypes [37]. These findings indicate that neither the strain nor the genotype is a determinant during the first contact between DENV and the mosquito vector. No statistically significant differences were found in the amounts of any of the DENV-3 genotype III strain viral genomes that were obtained from the infected mosquitoes, with the exception of the day $8 \mathrm{pf}$ time point, when a larger amount of the DENV-3/15859 strain genome was detected (Figure 4B). In terms of biological efficacy, these results suggest that the two isolates from the Medellin-Colombia area from the same serotype and viral genotype have the same potential for transmission at day $14 \mathrm{pf}$, the day on which previous reports suggested the conclusion of the incubation period in the vector or the extrinsic incubation period [38].
Although coinfections have not been sufficiently studied in both vectors and human hosts, existing evidence supports the idea that some serotypes can be found simultaneously in both mosquitos of the Aedes genus [22,23] and humans [18,19,21]. Coinfections of the vector are highly important, especially in endemic areas in which all four serotypes cocirculate, because the vector can support infections of two or more serotypes by feeding on two different individuals. This behavior would subsequently affect the viral transmission dynamics [22].

In the in vitro coinfection assessments with the DENV-2/3986 and DENV-3/3832 strains, the serotype 2 strain was found to replicate more efficiently than the serotype 3 strain (Figure 5). A similar behavior was observed in coinfections of $\mathrm{C} 6 / 36$ cells with serotype 2 and 4 strains. In this case, the levels of replication of DENV-2 were lower than those of DENV-4 [24]. These findings could indicate that under conditions of coinfections in cell lines, predominant replication of one serotype could be due to the presence of specific receptors that allow entry, as previously explained. For example, a lamininbinding protein has been described as being responsible for the entry of serotype 3 [33], while the entry of serotype 2 into mosquito cells has been linked to Hsc 70 proteins (shock cognate protein 70) [39] and prohibitin [32]. So far, mechanisms that relate to differences in replication once the virus has entered the cell have not been described.

The in vivo coinfection results also showed that the serotype 2 strain had higher replication efficiency (more than 1000 more viral genome copies than serotype 3; Figure 6). Although this is the first report of artificial coinfections, serotypes 2 and 3 are known to have simultaneously shared the same vector and host in Asia [22], a fact that is related to both the ability of both viruses to replicate efficiently in the vector and the ability of the vector to transmit the viruses. These factors have not been studied naturally. The findings obtained in this study are a first approach to the virus-vector relationship in the event of coinfection. The finding that serotype 2 replicated more efficiently than serotype 3 agrees with studies that have suggested that serotype 2 strains, mainly those of the Southeast Asian genotype, are more virulent than strains of other genotypes [29], a fact that might explain the replicative advantage of serotype 2 relative to serotype 3 . However, these differences are not only virus associated, because the vectors are known to have natural barriers that prevent midgut infection or spreading to the salivary glands [40]. 
Although there are few studies on the modulation of immune responses against DENV infections in the mosquito, serotype 2 is known to be able to suppress the toll-like receptor-mediated responses [41], which might favor the replication of serotype 2 over serotype 3.

Considering these results and although there are no known reports of natural coinfections in Colombia, the constant circulation of all four serotypes (serotypes 2 and 3 have been predominant in recent years), strain re-introductions or recurrences, and the presence of more efficient vectors for the transmission of some serotypes indicate that Colombia is susceptible to infections that could result in epidemiological profile changes and favor the occurrence of severe disease forms. Additionally, taking these data together with epidemiological data from the last 30 years in Colombia, there is a close relationship between serotype 2 replicative advantage over other serotypes, it having been reported in more than $40 \%$ of Colombian isolates [13]. Nevertheless, further studies are needed to determine how the two serotypes might interact within the same vector and how one serotype might affect the replication of the other.

\section{Acknowledgements}

This study was funded by COLCIENCIAS (Departamento Administrativo de Ciencia, Tecnología e Innovación). Project 111549326083.

\section{References}

1. Weaver SC, Reisen WK (2010) Present and future arboviral threats. Antiviral Res 85: 328-345.

2. Lin CY, Huang CH, Chen YH (2013) Classification of dengue: the clinical use of World Health Organization 2009 guideline. J Formos Med Assoc 112: 61-63.

3. Clyde K, Kyle JL, Harris E (2006) Recent advances in deciphering viral and host determinants of dengue virus replication and pathogenesis. J Virol 80: 11418-11431.

4. Bhatt S, Gething PW, Brady OJ, Messina JP, Farlow AW, Moyes CL, Drake JM, Brownstein JS, Hoen AG, Sankoh O, Myers MF, George DB, Jaenisch T, Wint GR, Simmons CP, Scott TW, Farrar JJ, Hay SI (2013) The global distribution and burden of dengue. Nature 496: 504-507.

5. Kanakaratne N, Wahala WMPB, Messer WB, Tissera HA, Shahani A, Abeysinghe N, de-Silva AM, Gunasekera M (2009) Severe dengue epidemics in Sri Lanka, 2003-2006. Emerg Infect Dis 15: 192-199.

6. Chaturvedi UC, Nagar R (2008) Dengue and dengue haemorrhagic fever: Indian perspective. J Biosci 33: 429-441.

7. Diallo M, Ba Y, Sall AA, Diop OM, Ndione JA, Mondo M, Girault L, Mathiot C (2003) Amplification of the sylvatic cycle of dengue virus type 2, Senegal, 1999-2000: entomologic findings and epidemiologic considerations. Emerg Infect Dis 9: 362-367.
8. Gómez-Dantés H, Willoquet JR (2009) Dengue in the Americas: challenges for prevention and control. Cad Saúde Pública 25 Suppl 1: S19-31.

9. Vasilakis N, Deardorff ER, Kenney JL, Rossi SL, Hanley KA, Weaver SC (2009) Mosquitoes put the brake on arbovirus evolution: experimental evolution reveals slower mutation accumulation in mosquito than vertebrate cells. PLoS Pathog 5: e1000467.

10. Craig S, Thu HM, Lowry K, Wang X, Holmes EC, Aaskov J (2003) Diverse dengue type 2 virus populations contain recombinant and both parental viruses in a single mosquito host. J Virol 77: 4463-4467.

11. King CC, Chao DY, Chien L-J, Chang GJJ, Lin TH, Wu YC, Huang JH (2008) Comparative analysis of full genomic sequences among different genotypes of dengue virus type 3 . Virol J 5: 63.

12. Ocazionez RE, Gómez SY, Cortés FM (2007) [Dengue hemorrhagic fever serotype and infection pattern in a Colombian endemic area]. Rev Salud Pública Bogotá Colomb 9: 262-274.

13. Méndez JA, Usme-Ciro JA, Domingo C, Rey GJ, Sánchez JA, Tenorio A, Gallego-Gomez JC (2012) Phylogenetic reconstruction of dengue virus type 2 in Colombia. Virol J 9: 64.

14. Rodriguez-Roche R, Alvarez M, Holmes EC, Bernardo L, Kouri G, Gould EA, Halstead S, Guzman MG. (2005) Dengue virus type 3, Cuba, 2000-2002. Emerg Infect Dis 11: 773-774.

15. Rico-Hesse R (2007) Dengue virus evolution and virulence models. Clin Infect Dis Off Publ Infect Dis Soc Am 44: 14621466.

16. Villabona-Arenas CJ, Miranda-Esquivel DR, Jimenez RE (2009) Phylogeny of dengue virus type 3 circulating in Colombia between 2001 and 2007. Trop Med Int Health 14: 1241-1250.

17. Rico-Hesse R (1990) Molecular evolution and distribution of dengue viruses type 1 and 2 in nature. Virology 174: 479-493.

18. Gupta E, Dar L, Broor S (2008) Concurrent infection by two dengue virus serotypes among dengue patients. Indian J Med Microbiol 26: 402-403.

19. Pongsiri P, Themboonlers A, Poovorawan Y (2012) Changing pattern of dengue virus serotypes in Thailand between 2004 and 2010. J Health Popul Nutr 30: 366-370.

20. Figueiredo RMP de, Naveca FG, Oliveira CM, Bastos M de S, Mourão MPG, Viana Sde S, Melo Mdo N, Itapirema EF, Saatkamp CJ, Farias IP (2011) Co-infection of Dengue virus by serotypes 3 and 4 in patients from Amazonas, Brazil. Rev Inst Med Trop São Paulo 53: 321-323.

21. Mamani E, Figueroa D, García MP, Garaycochea M del C, Pozo EJ (2010) [Concurrent infections by two dengue virus serotypes during an outbreak in northwestern Peru, 2008]. Rev Peru Med Exp Salud Pública 27: 16-21.

22. Thavara U, Siriyasatien P, Tawatsin A, Asavadachanukorn P, Anantapreecha S, Wongwanich R, Mulla MS (2006) Double infection of heteroserotypes of dengue viruses in field populations of Aedes aegypti and Aedes albopictus (Diptera: Culicidae) and serological features of dengue viruses found in patients in southern Thailand. Southeast Asian J Trop Med Public Health 37: 468-476.

23. Martins VEP, Alencar CH, Kamimura MT, Kamimura MT, de Carvalho Araújo FM, De Simone SG, Dutra RF, Guedes MI (2012) Occurrence of natural vertical transmission of dengue- 2 and dengue-3 viruses in Aedes aegypti and Aedes albopictus in Fortaleza, Ceará, Brazil. PloS One 7: e41386. 
24. Pepin KM, Hanley KA (2008) Density-dependent competitive suppression of sylvatic dengue virus by endemic dengue virus in cultured mosquito cells. Vector Borne Zoonotic Dis 8: 821828.

25. Hanley KA, Nelson JT, Schirtzinger EE, Whitehead SS, Hanson CT (2008) Superior infectivity for mosquito vectors contributes to competitive displacement among strains of dengue virus. BMC Ecol 8: 1.

26. Vazeille M, Mousson L, Martin E, Failloux AB (2010) Orally co-Infected Aedes albopictus from La Reunion Island, Indian Ocean, can deliver both dengue and chikungunya infectious viral particles in their saliva. PLoS Negl Trop Dis 4: e706.

27. Ospina MC, Diaz FJ, Osorio JE (2010) Prolonged cocirculation of two distinct Dengue virus Type 3 lineages in the hyperendemic area of Medellin, Colombia. Am J Trop Med Hyg 83: 672-678.

28. Chien LJ, Liao TL, Shu PY, Huang JH, Gubler DJ, Chang GJ (2006) Development of real-time reverse transcriptase PCR assays to detect and serotype dengue viruses. J Clin Microbiol 44: 1295-1304.

29. Anderson JR, Rico-Hesse R (2006) Aedes aegypti vectorial capacity is determined by the infecting genotype of dengue virus. Am J Trop Med Hyg 75: 886-892.

30. Chomczynski P, Sacchi N (1987) Single-step method of RNA isolation by acid guanidinium thiocyanate-phenol-chloroform extraction. Anal Biochem 162: 156-159.

31. Rodríguez-Roche R, López Matilla L, Alvarez Vera M, Morier Díaz L, Guzmán Tirado MG (2012) [Biological properties of virus dengue-3 strains isolated during the epidemic ocurred in Havana, 2001-2002]. Rev Cubana Med Trop 64: 187-198.

32. Kuadkitkan A, Wikan N, Fongsaran C, Smith DR (2010) Identification and characterization of prohibitin as a receptor protein mediating DENV-2 entry into insect cells. Virology 406: 149-161.

33. Sakoonwatanyoo P, Boonsanay V, Smith DR (2006) Growth and production of the dengue virus in C6/36 cells and identification of a laminin-binding protein as a candidate serotype 3 and 4 receptor protein. Intervirology 49: 161-172.

34. Hidari KIPJ, Suzuki T (2011) Dengue virus receptor. Trop Med Health 39: 37-43.
35. Rodríguez-Roche CR, López Matilla L, Alvarez Vera CM, Morier Díaz L, Guzmán Tirado CMG (2011) [Dengue virus serotype 2 strains isolated from the epidemic occurred in Santiago de Cuba in 1997]. Rev Cubana Med Trop 63: 211219.

36. Maciel-De-Freitas R, Codeço CT, Lourenço-De-Oliveira R (2007) Body size-associated survival and dispersal rates of Aedes aegypti in Rio de Janeiro. Med Vet Entomol 21: 284292.

37. Cox J, Brown HE, Rico-Hesse R (2011) Variation in vector competence for dengue viruses does not depend on mosquito midgut binding affinity. PLoS Negl Trop Dis 5: e1172.

38. Salazar MI, Richardson JH, Sánchez-Vargas I, Olson KE, Beaty BJ (2007) Dengue virus type 2: replication and tropisms in orally infected Aedes aegypti mosquitoes. BMC Microbiol 7: 9.

39. Paingankar MS, Gokhale MD, Deobagkar DN (2010) Dengue-2-virus-interacting polypeptides involved in mosquito cell infection. Arch Virol 155: 1453-1461.

40. Bennett KE, Olson KE, Muñoz M de L, Fernandez-Salas I, Farfan-Ale JA, Higgs S, Black WC 4th, Beaty BJ (2002) Variation in vector competence for dengue 2 virus among 24 collections of Aedes aegypti from Mexico and the United States. Am J Trop Med Hyg 67: 85-92.

41. Sim S, Dimopoulos G (2010) Dengue virus inhibits immune responses in Aedes aegypti cells. PloS One 5: e10678.

\section{Corresponding author}

Marlén Martínez-Gutierrez

Grupo de Investigación para el Fortalecimiento de la Salud y el Bienestar-GIFOSABI.

Universidad Cooperativa de Colombia, Calle 30A \# 33-51

Bucaramanga, Colombia.

Phone: (57+7) 6327424

Fax Number: $(57+7) 6327424$

Email: marlen.martinezg@campusucc.edu.co

Conflict of interests: No conflict of interests is declared. 